\title{
A Study on the Financing Efficiency of Small and Medium-sized Enterprises Assemble Bond Based on DEA
}

\author{
Weizhang Li, Chi Xie \\ College of Business Management Hunan University, Changsha, Hunan Province, China \\ LiWeizhang54@163.com,xiechi@hnu.edu.cn
}

\begin{abstract}
The assemble bond is a new innovative financing channel for small and medium-sized enterprises (SMEs), which has unparalleled advantages in solving the financing difficulty. At first, to construct the SMEs assemble bond and board financing efficiency indicator system on the basis of DEA model. Then apply this model to make an empirical study. And the empirical result reflects that the SMEs assemble bond's financing efficiency is higher than the SMEs board. In addition, we will investigate the advanced way to improve the financing efficiency of the SMEs assemble bond, which is to adjust the rate of financing cost and the main business revenue growth.
\end{abstract}

Index Terms - DEA, SMEs assemble bond, financing efficiency.

\section{Introduction}

The small and medium-sized enterprise (SMEs) play an active role in promoting national economic growth, solving the employment and so on for ages, which has become an indispensable important part of economic and social development. However, the problem of funds short has been severely hampered the healthy development of SMEs. As one of the major financing way of SMEs, the debt financing also has the plight of low efficiency: it means that the SMEs will be in the weakly position because of the scale of these corporations is smaller than the huge companies and the risk is higher which should be taken. As a result, these organizations have to face numerous barriers in the financing process. Moreover, the previous relevant research states that the financing process of SMEs is lower in China. In recent years, the SMEs assemble bond as the bond financing market innovative product which has been published in the financial market of China. And it provides a new way to resolve the bond financing problem of SMEs.

The SMEs' assemble bond refers to the SEMs as the publishing main body a number of SMEs issuance bond in the form of assemble, forming a general distribution limit and using a uniform name. A reasonable structure and proper number of assemble bond can improve the enterprise governance, and promote the enterprise development in a suitable way to improve the financing efficiency of SMEs. In addition, the advantage of bond is lowing financing process cost and this process speed is more quickly than old ways. It means that the SMEs assemble bond will be popular in the financial markets. Moreover, the financing efficiency is extremely important for the enterprise development. As a result, to apply the scientific evaluation on the financing efficiency of SMEs assemble bond is significant.

SMEs financing efficiency evaluation can be summarized as two ways. The first one is the fuzzy comprehensive evaluation. Zhu [1] used the fuzzy comprehensive evaluation method to analyse Zhejiang province's SMEs, and he concludes that the efficiency of bond financing is higher than the equity financing efficiency. Tian [2] applied the fuzzy comprehensive evaluation method to evaluate the different financing ways of China enterprise group and put forward corresponding suggestions on the basis of the enterprise group financing characteristics analysis. Another way is to build a data envelopment analysis (DEA) model with calculation indicators input to evaluate SMEs' financing efficiency. Zeng and Chen [3] employed the DEA method from the aspect of debt financing cost and integrated capital using cost to evaluate the financing efficiency of SMEs. And they apply the CCR model of DEA to analyse the sample. Wang and Huang [4] proposed the combination debt financing efficiency evaluation method of cluster analysis and DEA. It is clearly defining the intension and extension meaning of the debt financing efficiency and building the corresponding evaluation indicator system. Zeng and Zhou [5] built a SMEs financing efficiency evaluation model based on the DEA and analyse SMEs assemble bond, listing, folk financing and bank loans' financing efficiency contrastively. They consider that the SMEs assemble bond is irreplaceability and has a significant difference with other three ways. Liu and Wang [6] researched the data of 27 list companies which issued bonds though nonparametric super efficiency hybrid DEA and stated that the overall sample is in a relatively low efficiency. Huang [7] respectively operated the DEA and super efficiency DEA evaluation methods to analyse the SMEs assemble bond, board and GEM (growth enterprise market) financing efficiency. And to discuss several factors that influence the financing efficiency of SMEs.

In the end, we reflect that numerous scholars would like to choose the DEA and fuzzy comprehensive methods to study SMEs financing efficiency. However, the expert scoring in fuzzy comprehensive has a strong subjective dependency, which is lack of objective persuasive on the contrast of DEA, because the DEA depends on data accuracy.

The research work was supported by the National Natural Science Foundation of China under Grant No. 71373072, the Specialized Research Fund for the Doctoral Program of Higher Education under Grant No. 20130161110031, the Hunan Provincial Innovation Foundation for Postgraduate under Grant No. CX2013A006, and the Foundation for Innovative Research Groups of the National Natural Science Foundation of China under Grant No. 71221001. 
Therefore, this article mainly uses the CCR model in DEA (the basic DEA model proposed by Charnes, Cooper and Rhode [8]) to study SMEs assemble bond' financing efficiency and discover the suitable way to improve the SMEs financing efficiency.

\section{Construction of Evaluation Model}

The DEA is a goal programming method based on the concept of relative efficiency. It puts all the good efficiency Decision Making Units (DMU) together to form a production frontier, and other units of relatively poor efficiency fall within the efficiency boundary.

The DEA model is based on the multiple input and single output models: Farrell [9] reflected that applying the production frontier or efficiency frontier to measure productive, and replaces "the Default Production Function" by "the Preset Production Function". Furthermore, all DMUs are regarded as the same production function and apply the broken line to connect the appropriate DMU points to establish an envelope line to assess the efficiency. And do not need to estimate any parameters in this model. If the DMU point is in the efficient frontier border, it illustrates that the DMU is effective and the efficiency value is 1 .

In addition, if the point is within the efficiency frontier border, it means that the DMU is relatively inefficient and the value is between 0 and 1 . This model has been continuous improved and corrected which is introduced by Farrell [9]. Currently, the model has been developed into CCR model which means "multiple inputs and multiple outputs". It is more adaptable to the increasingly complex business processes.

To suppose the input indicator of SMEs' financing efficiency evaluation indexes are $x_{1 j}, x_{2 j}, x_{3 j}, j=\{1,2, \ldots, n\}$, and output indicators are $y_{1 j}, y_{2 j}, y_{3 j}, j=\{1,2, \ldots, n\}$. Moreover, the evaluation model of SMEs' financing efficiency could be constructed by the CCR model of DEA as follow:

$$
\left\{\begin{array}{l}
\max _{u, v}\left(u^{T} y_{j} / v^{T} X_{J}\right) \\
\text { s.t. }\left\{\begin{array}{l}
\left(u^{T} y_{j} / v^{T} X_{J}\right) \leq 1, j=1, \ldots, n \\
u \geq 0, v \geq 0
\end{array}\right.
\end{array}\right.
$$

Because (1) is a fractional program, and it is inconvenience to solve and may appear multiple solutions or infinite solutions. Therefore, (1) should be transformed into multivariate linear programming model. And apply the $\omega$ to represent the efficiency value of the $j$ decision making unit, which satisfied $0 \leq \omega \leq 1$. The $x_{i, j}$ and $y_{r, j}$ means that the $j$ decision making unit's the $i$ input and the $j$ output.

It is necessary to add the Archimedes Dimensionless $\varepsilon$ ( $\varepsilon$ is a number greater than 0 and less than any positive number) and slack variables $s_{i}^{-}$and $s_{r}^{+}$in order to regulate variables better. Then we can get (2) where $s_{i}^{-}$and $s_{i}^{+}$are equal to the difference between the input $x$ and output $y$.
The CCR model considers that when $\omega=1$ and $s_{i}^{-}=s_{r}^{+}=0$, the decision-making unit is efficient, otherwise it is weak efficient or inefficient relatively to other decision making units.

$$
\left\{\begin{array}{l}
\min \left[\omega-\varepsilon\left(\sum_{i=1}^{3} s_{i}^{-}+\sum_{r=1}^{3} s_{r}^{+}\right)\right] \\
\text {s.t. }\left\{\begin{array}{l}
\sum_{j=1}^{n} x_{i, j} \lambda_{j}+s_{i}^{-}=\omega x_{i j}(i=1,2,3) \\
\sum_{j=1}^{n} x_{r j} \lambda_{j}-s_{r}^{+}=y_{r j}(r=1,2,3) \\
\omega, \lambda, s_{i}^{-}, s_{r}^{+} \geq 0, j=1,2, \ldots, n
\end{array}\right.
\end{array}\right.
$$

\section{Indictors System Determination and Sample Selection}

\subsection{Determination of Calculating Indicators Based on DEA}

In general, the financing efficiency of enterprises mainly reflects in two aspects: efficiency financing and integrated capital allocation efficiency. Zeng and Zhou [5] stated that the efficiency financing of SMEs should consider the comparative analysis of financing cost, financing risk, capital allocation efficiency and management quality (includes the profitability, growth ability, capital operation ability and others). In addition, the scale of enterprise, the fixed assets ratio and corresponding financial index are important indicators of SMEs' financing efficiency.

As a consequence, this paper summarises the SMEs' financing efficiency evaluation indicator system based on the existing literature as shown in Table 1, and there are two aspects in this evaluation system: the input indicators and the output indicators. Furthermore, it includes six variables.

TABLE1: SMEs' Financing Efficiency Evaluation Indictors Based on the DEA

\begin{tabular}{|l|c|c|}
\hline \multirow{4}{*}{$\begin{array}{l}\text { Input } \\
\text { indicators }\end{array}$} & Indicator type & Indicator variables \\
\cline { 2 - 3 } & Financing cost & Financing cost rate \\
\hline \multirow{3}{*}{$\begin{array}{c}\text { Output } \\
\text { indicators }\end{array}$} & Profitability & Asset-liability ratio \\
\cline { 2 - 3 } & Growth ability & Quick ratio \\
\cline { 2 - 3 } & Capital operation ability & Return on total assets rate \\
\hline
\end{tabular}

The input indicator reflects the enterprise financing efficiency. It is mainly expressed by the corresponding cost in the financing process. The bond issuing party will charge relevant fee according to the SMEs' condition. In addition, when an enterprise chooses different bond portfolios, the capital cost, financing cost and opportunity cost will be different. Consequently, to apply the enterprise financing cost rate, asset-liability ratio and quick ratio to measure the efficiency in the financing process. 
The output indicators mainly state the usage efficiency of capital integrated. It means that the usage efficiency of capital integrated is the ability of enterprise uses the capital effectively. The more the funds is in place, the higher the financing efficiency is. And it is usually reflecting in enterprise profit ability, growth ability and capital operation ability. It states that the company which has strong profitability and own strong debt paying ability, the investor would like to invest more capital, and companies financing efficiency will increase accordingly. Additional, the growth ability of enterprise is positively related to the financing efficiency. Because of some high growth ability organizations have great potential development ability to attract venture capital to promote the enterprise development and improve the corporate financing efficiency. Besides, the capital operation ability is a positive correlation relationship with the financing efficiency. It is obvious that the stronger the operation ability is and the faster the capital turnover speeds is, the higher the financing efficiency will be.

\subsection{Sample Selections}

To ensure the result is objective accuracy, this paper according to the definition of SMEs in "The SMEs promotion law of the People's Republic of China" to determine the research object. It selects enterprises which main business revenues or total assets is less than RMB 500 million and the number of employees is less than 3000 corporations as the sample. More exactly, to eliminate the ST enterprises, enterprises with abnormal extreme value and the enterprise whose financial disclosure is not complete because of the extreme value will influence on statistical results.

This article selects the assemble bonds of 16 SMEs which issues in 2011 as the research decision-making unit. And we choose 26 enterprises of the same industry as the reference sample from the SMEs board to eliminate effects from macro environment factors and industry factors on the results and consider the input and output hysteresis. At last, there are 42 enterprises as sample in this paper. The number of the sample meets the requirement of DEA model: firstly, the number of samples is larger than the number of input variables multiplies the number of output variables. Secondly, the number of samples is larger than three times of the sum of the number of input variables plus the number of output variables.

\section{Empirical Results and Analysis}

\subsection{Descriptive Statistical Analysis}

This article uses the Matlab 7.5 software programming to calculate the sample enterprises' corresponding financing efficiency value through the SMEs financing efficiency CCR evaluation model of DEA constructed above in order to evaluate the SMEs' assemble bond and board' financing efficiency. The evaluation's descriptive statistics results are presented in Table 2 .
TABLE 2: Sample Enterprises’ Financing Efficiency’s Descriptive Statistics

\begin{tabular}{|c|c|c|c|c|c|}
\hline SMEs & Maximum & Minimum & Mean & Medium & S.D. \\
\hline Assemble bond & 1 & 0.1315 & 0.697 & 0.8073 & 0.3433 \\
\hline Board & 1 & 0.2735 & 0.6315 & 0.5148 & 0.2612 \\
\hline
\end{tabular}

From Table 2, we can see the maximum of SMEs assemble bond financing efficiency is 1 , the minimum value is 0.1315 , the median is 0.8073 and the standard deviation is 0.3433 , which show that the financing efficiency of SMEs assemble bond varies with different enterprises. The financing efficiency' mean of SMEs assemble bond is higher than the SMEs board, which illustrates that SMEs assemble bond financing efficiency is high.

\subsection{Financing Efficiency's Effectiveness Analysis}

As be mentioned above, $\omega$ is a financing efficiency value of decision making units, and $s_{r}^{+}, s_{t}^{-}$are slack variables, respectively represent input redundancy rate and output inadequate rate. The DEA method's effectiveness determination standard and economic significance is as follow: (1) When $\omega=1$ and $s_{r}^{+}=s_{t}^{-}=0$, it illustrates that the decision making unit is effective, that is to say this decision making unit achieve the optimal output $Y$ on the basis of the input $X$. Among them, the $X$ and $Y$ respectively represent the set of input and output indicators. (2) When $\omega=1$ and $s_{r}^{+} \neq 0$, $s_{t}^{-} \neq 0$, it can be determined the decision making unit is weak efficient, which means that the decision making unit can reduce part of the input $X$ at the same time keep the original output $Y$ constant, or can keep input $X$ constant then increase output $Y$. (3) When $\omega<1$, it can be determined the decision making unit is invalid, which means the improper input of decision making unit should be geometric compressed comprehensively.

According to above judgment standards, one of this paper' sample enterprise Kaifeng Tenai limited company' financing efficiency value $\omega=0.8073$, so it indicates that the enterprise financing efficiency is invalid. In addition, $s_{1}^{-}=0.0024, s_{1}^{+}=0.2635$, correspondingly it means that financing cost rate has redundancy and the main business revenue growth rate is insufficient, therefore this enterprise can adjust the financing efficiency from reducing financing cost and improving the main business income. The statistical situation of sample enterprises' financing efficiency value is organized in Table 3.

TABLE 3: Effectiveness Comparison of Sample Enterprise' Financing Efficiency

\begin{tabular}{|c|c|c|c|c|c|c|}
\hline \multirow{2}{*}{ SMEs } & \multicolumn{2}{|c|}{ Effective } & \multicolumn{2}{c|}{ Weak effective } & \multicolumn{2}{c|}{ Invalid } \\
\cline { 2 - 7 } & Number & Rate & Number & Rate & Number & Rate \\
\hline $\begin{array}{c}\text { Assemble } \\
\text { bond }\end{array}$ & 7 & $43.75 \%$ & 0 & 0 & 9 & $56.25 \%$ \\
\hline Board & 4 & $15.38 \%$ & 0 & 0 & 22 & $84.62 \%$ \\
\hline
\end{tabular}


The effectiveness comparison of sample enterprise' financing efficiency value results show that the effectiveness ratio of the SMEs assemble bond is $43.75 \%$, far higher than the SMEs board; both of this two kinds financing ways have no weak effective enterprises. It also shows that using assemble bond financing for SMEs has higher effectiveness. Assemble bond as a new financing way, has a very good development prospect.

Because through the slack variables of SMEs assemble bond' financing efficiency we can know the place and the directions needed to be noticed to improve sample enterprises' financing efficiency, so in this paper we carry on a statistical analysis of the slack variables, and find that most of the non-zero input indictor of variable $s_{1}^{-}$is financing cost, and the non-zero companies account for $37.5 \%$; as for variable $s_{1}^{+}$, most of the non-zero output indictor is the main business revenue growth, and the nonzero companies percentage is $43.75 \%$. It can be concluded that the key factors influence SMEs assemble bond' financing efficiency are the financing cost and main business revenue growth. It may be that the SMEs financing is difficult and hard to control its financing cost, even if issue assemble bond, the financing cost is also an important factor influencing the financing efficiency. Main business revenue growth mainly reflects the enterprise product life cycle, and SMEs' new product development is difficulty, so it seriously affect the assemble bond financing efficiency.

\section{Conclusions}

The SMEs assemble bond plays a positive role in improving the financing environment and broadening the financing channels of SMEs in China. The overall financing efficiency and effectiveness of SMEs is higher than the SMEs board, so it is visible that SMEs assemble bond has good development potential and market prospects. Because different enterprises have different financing efficiency values, so SMEs should be careful to choose suitable financing ways. The final empirical results also find that the financing cost rate and the main business revenue growth are main factors that influence financing efficiency of SMEs assemble bond commonly. In order to make the whole of the assemble bond financing is more efficient, the SMEs should pay more attention to reducing financing cost rate and improving the main business revenue growth when issuing assemble bond.

\section{References}

[1] B.-X. Zhu, "Zhejiang small and medium-sized enterprise financing efficiency's fuzzy comprehensive evaluation and empirical analysis," Zhejiang Statistics, vol.10, pp.12-15, 2005.

[2] F. Tian, "The enterprise group finance efficiency comparison based on the multiple factors," Statistics and Decision, vol.8, pp.179-182, 2011.

[3] J.-H. Zeng and D.Y. Chen, "A Study on the financing efficiency of small and medium-sized enterprises debt based on DEA," Economic Theory and Business Management, vol.1, pp.50-53, 2008.

[4] X.-H. Wang, L.-N. Huang, and L.N. Huang, "A Study on the efficiency evaluation index system of enterprise debt financing based on the DEA," Journal of Inner Mongolia Finance and Economics College, vol.2, pp.62-65, 2010.

[5] J.-H. Zeng and Y. Zhou, "Research on small and medium-sized enterprise assemble bond financing efficiency," Seeker, vol.1, pp.21$23,2010$.

[6] C. Liu and B. Wang, "A study on issued companies bonds of listed companies financing efficiency evaluation based on the mixed DEA model," Business Economy, vol.4, pp.76-78, 2010.

[7] W.-F. Huang, Research on our country small and medium-sized enterprise assemble bond's financing efficiency: [North university of China master's degree thesis]. Taiyuan: Economics and management institute of North university of China, 2013, 22-30.

[8] A. Charnes, W.W. Cooper and E. Rhodes, "Measuring the efficiency of decision making units," European Journal of Operational Research, vol.2, no.6, pp.429-444, 1978.

[9] M.J. Farrell, "The measurement of productive efficiency," Journal of Royal Statistical Society, vol.120, no.3, pp.253-281, 1957. 